\author{
C.M. BовK
}

\title{
МЕТОДЫ ЧИСЛЕННОЙ МИНИМИЗАЦИИ ФУНКЦИОНАЛА КВАЗИПРОТЯЖЕННОСТИ
}

Аннотация. Предложены методы численной минимизации функционала квазипротяжности, который построен на основе функции невязки между данными и их моделью. Эти методы включают методы одномерной минимизации по неизвестному параметру модели данных, который входит в модель данных линейно или нелинейно и принимает одно или несколько значений, и метод многомерной минимизации по нескольким неизвестным линейным параметрам модели, каждый из которых принимает одно значение. Методы одномерной минимизации основаны на методах оптимизации нулевого порядка. Метод многомерной минимизации основан на методе сопряженных градиентов. Приведены примеры численного моделирования, которые демонстрируют эффективность использования предложенных методов.

Ключевые слова: функционал, минимизация, моделирование.

Введение. В [1] приведены постановки задач обработки данных, которые основаны на критерии минимума протяженности [2]. Успешное решение этих задач зависит от качества настройки функционала квазипротяженности [3] и от качества его численной минимизации. В данной работе рассмотрены методы численной минимизации функционала квазипротяженности для случая, когда он построен на основе функции невязки между данными и их моделью. Эти методы включают в себя методы одномерной минимизации и метод многомерной минимизации. Методы одномерной минимизации предназначены для решения задачи определения значений одного неизвестного параметра, который входит в модель данных линейно или нелинейно и принимает одно или несколько значений. Метод многомерной минимизации предназначен для решения задачи определения значений нескольких неизвестных линейных параметров модели, каждый из которых принимает одно значение.

(C) Вовк С.М., 2019 
«Системні технології» 5 (124) 2019 «System technologies»

Постановка задачи и цель исследований. Постановка задачи заключается в разработке методов одномерной и многомерной численной минимизации функционала квазипротяженности, который построен на основе функции невязки между данными и их моделью. Целью данной работы является создание эффективных методов численной минимизации функционала квазипротяженности.

Анализ последних исследований и публикаций. Согласно [4], протяженность абстрактной функции $f(x)$ определяется функционалом:

$$
E[f(x)]=\int_{-\infty}^{\infty} \chi[f(x)] d x
$$

где функция $\chi[f(x)]=\left\{\begin{array}{ll}1 ; & f(x) \neq 0 \\ 0 ; & f(x)=0\end{array}\right.$ играет роль идеальной стоимостной функции. Если $f(x)$ задает функцию отклонения исходных данных от их модели, то минимизация (1) означает минимизацию протяженности области несовпадения исходных данных с их моделью [2].

Обобщением (1) является функционал квазипротяженности [2]

$$
E^{(\alpha, \beta, q)}[f(x)]=\int_{-\infty}^{\infty} \psi_{S}^{(\alpha, \beta, q)}[f(x)] d x
$$

в котором стоимостная функция $\psi_{S}^{(\alpha, \beta, q)}$ имеет вид [1]:

$$
\psi_{S}^{(\alpha, \beta, q)}(x)=k_{S}^{(\alpha, \beta, q)}\left[\left(1+|x / \alpha|^{q}\right)^{\beta / q}-1\right],
$$

где $\alpha$ - параметр сглаживания; $q$ - параметр степени сглаживания, причем $0<q<\infty ; \quad \beta$ - параметр формы, причем $-\infty<\beta \leq 1$ и $\beta<q$; $k_{S}^{(\alpha, \beta, q)}=1 /\left[\left(1+\left|x_{0} / \alpha\right|^{q}\right)^{\beta / q}-1\right] ; x_{0}$ - точка нормировки (3) на единицу [2]. Параметры $\alpha, \beta, q$ имеют смысл свободных параметров и позволяют менять поведение функции (3). В [3] показано, что эта функция обеспечивает широкие возможности по настройке процесса обработки данных на текущую шумовую обстановку.

Критерий минимума протяженности заключается в требовании минимизировать протяженность функции, используемой для поиска решения [2]. Применение этого критерия к задаче с одним неизвестным линейным параметром приводит к ее формулировкам в виде [2]:

$$
\min _{A} E[g(x)-A f(x)]
$$


«Системні технологіï» 5 (124) 2019 «System technologies»

$$
\min _{A} E^{(\alpha, \beta, q)}[g(x)-A f(x)],
$$

где функция $g(x)$ описывает исходные данные, $A$ есть неизвестный линейный параметр, а функция $f(x)$ задает известную форму модели данных. Отличие (4) от (5) заключается в том, что постановка (4) применима только к ограниченным по аргументу и свободным от шума функциям, тогда как область применимости постановки (5) является более широкой за счет включения в нее как зашумленных функций, так и функций, почти ограниченных по аргументу. Кроме того, постановка (5) включает постановку (4) в качестве своего частного случая [2].

Анализ свойств функционала квазипротяженности $E^{(\alpha, \beta, q)}$ показывает [3], что для его минимизации невозможно применить аналитические методы вариационного исчисления [5], а также стандартные методы численной оптимизации [6]. Это обусловлено тем, что данный функционал не является выпуклым (за исключением некоторых крайних случаев), а его первая вариация в точке минимума может не существовать. Однако практическая применимость функционала квазипротяженности обеспечивается параметризацией модели в совокупности с дискретизацией исходных зависимостей и представлением их в виде конечного набора числовых данных. Как известно [5], параметризация модели является одним из ключевых моментов построения минимизирующей последовательности для численного решения вариационных задач. С ее помощью решение вариационной задачи сводится к задаче минимизации целевой функции, которая зависит от конечного числа параметров, а не от бесконечного числа значений варьируемой функции. Для случая нескольких линейных параметров это достигается путем представления искомого решения в виде суммы известных линейно независимых «координатных» функций с неизвестными числовыми коэффициентамипараметрами, которые находят посредством минимизации соответствующего функционала. Однако в отличие от традиционно используемого для этой цели квадратичного функционала [5], функционал квазипротяженности предоставляет возможность решения задач, в которых исходные данные искажены грубыми ошибками, а каждый линейный параметр может принимать не одно, а несколько значений [3]. 
«Системні технологіï» 5 (124) 2019 «System technologies»

Основная часть. Рассмотрим задачу (5) одномерной минимизации функционала квазипротяженности для случая линейного параметра. Развернутая запись этой задачи для дискретного случая есть:

$$
\min _{A} k_{S}^{(\alpha, \beta, q)} \sum_{n=1}^{N}\left[\left(1+\left|\left(g_{n}-A f_{n}\right) / \alpha\right|^{q}\right)^{\beta / q}-1\right]
$$

где $g_{n}=g\left(x_{n}\right), f_{n}=f\left(x_{n}\right), x_{n}=n \cdot \Delta x, \Delta x$ - известный шаг дискретизации аргумента $x$. Важно отметить, что для $0<\beta<1$ имеем $k_{S}^{(\alpha, \beta, q)}>0$, для $-\infty<\beta<0$ имеем $k_{S}^{(\alpha, \beta, q)}<0$, а для $\beta \rightarrow \pm 0$ из (6) получаем задачу:

$$
\min _{A} k_{S}^{(\alpha, 0, q)} \sum_{n=1}^{N} \ln \left[1+\left|\left(g_{n}-A f_{n}\right) / \alpha\right|^{q}\right]=\min _{A} \sum_{n=1}^{N} \ln \left[1+\left|\left(g_{n}-A f_{n}\right) / \alpha\right|^{q}\right],
$$

где $k_{S}^{(\alpha, 0, q)}=1 / \ln \left[1+\left|x_{0} / \alpha\right|^{q}\right]>0$. Из (6) и (7) видно, что в общем случае для произвольных значений своих свободных параметров эти задачи являются много экстремальными (мультимодальными) задачами оптимизации одномерной целевой функции относительно неизвестного параметра $A$. Действительно, пусть $\alpha>0,0<q<1$ и $-\infty<\beta<q$. Тогда для каждого значения $g_{n} / f_{n}$ на оси значений неизвестного параметра $A$ целевая функция, стоящая под знаком операции минимизации в (6) или в (7), будет иметь остроконечный локальный минимум с бесконечным разрывом в нем первой производной, который не исчезает при его наложении на гладкие участки других локальных минимумов. Следовательно, в этом случае целевая функция будет иметь от одного (если все значения $g_{n} / f_{n}$ одинаковые) до $N$ (если все значения $g_{n} / f_{n}$ разные) локальных минимумов. С другой стороны, если $\alpha \rightarrow \infty$ и $q>1$, то соответствующая целевая функция становится выпуклой и имеет единственный глобальный минимум [3]. Однако для практически важных случаев, когда $0<\alpha<\infty$, $-\infty<\beta<1$ и $1 \leq q<\infty$ (в частности, когда $0<\alpha<\infty, \beta=0, q=2$ [7] или $0<\alpha<\infty, \beta=0, q=1$ [8]), целевая функция функционала квазипротяженности является выпуклой только в окрестностях локальных минимумов, но не за их пределами. Таким образом, решение задачи одномерной минимизации функционала квазипротяженности для случая неизвестного линейного параметра полезно получать на основе методов оптимизации нулевого порядка. 
«Системні технологіï» 5 (124) 2019 «System technologies»

Аналогичный вывод справедлив и при применении модели с одним неизвестным нелинейным параметром $\theta$. В самом деле, подставляя в (6)-(7) $s_{n}(\theta)$ вместо $A f_{n}$, где $s_{n}(\theta)$ есть дискретное значение известной модельной функции $s(x ; \theta)$ в точке $x=x_{n}$, и указывая на минимизацию по параметру $\theta$, получим постановку задачи одномерной минимизации целевой функции функционала квазипротяженности по нелинейному параметру $\theta$. Из нее видно, что для тех значений параметра $\theta$, которые обращают в нуль модуль невязки $\left|g_{n}-s_{n}(\theta)\right|$, на графике соответствующей целевой функции могут возникнуть локальные минимумы. В силу нелинейности параметра $\theta$, эти локальные минимумы могут возникнуть даже в случае тех значений свободных параметров, для которых аналогичная целевая функция относительно линейного параметра является выпуклой.

Важной причиной мультимодальности целевой функции одного неизвестного параметра является также факт принятия им на интервале наблюдения не одного, а двух или более значений. Это может приводить к появлению на графике целевой функции нескольких локальных минимумов, которые могут оказаться даже равноправными по своей глубине. Учитывая такую возможность, к методам одномерной минимизации целевой функции, получаемой на основе функционала квазипротяженности, целесообразно предъявить требование поиска нескольких локальных минимумов с отбором среди них некоторого множества основных локальных минимумов.

Методы оптимизации нулевого порядка представляют собой самый общий подход к решению задач оптимизации, требуя для своей работы значений только минимизируемой функции. Важным условием их применения является удачный выбор допустимой области аргумента целевой функции. Из общих соображений можно указать, по крайней мере, два способа такого выбора: 1) задать область значений аргументапараметра априорно; 2) задать область значений аргумента-параметра апостериорно. Первый способ является целесообразным в том случае, когда есть априорные сведения о диапазоне значений неизвестного параметра и их точности. Примером его реализации является сетка значе- 
«Системні технології» 5 (124) 2019 «System technologies»

ний частоты в прямом дискретном преобразовании Фурье, когда априорно накладываются ограничения на наинизшую ненулевую и наивысшую частоты синусоид, которые могут содержаться в исходных данных. Второй способ является целесообразным в том случае, когда таких априорных сведений нет. В его рамках следует получить множество "пробных" значений неизвестного параметра путем решения линейного или нелинейного уравнения, описывающего заданную модель данных, относительно каждого элемента исходных данных и использовать полученное множество пробных значений для решения задачи минимизации (пример такого способа приведен в [9]). Модифицированная версия этого способа заключается в том, чтобы на основе множества пробных значений установить статистическими методами диапазон значений неизвестного параметра и задать точность поиска его значений. Одним из таких широко используемых методов установления диапазона значений неизвестного параметра является метод межквартильного интервала [10]. Этот метод в подавляющем большинстве случаев обеспечивает удачный выбор искомого диапазона, а точность поиска положения минимума задается априорно путем деления длины полученного диапазона на $10 . . .1000$ частей [11].

Поскольку рассматриваемая одномерная задача является мультимодальной, то метод ее минимизации должен находить все локальные минимумы. Поиск всех локальных минимумов можно осуществить следующим образом. Пусть область значений аргумента-параметра целевой функции представлена множеством из $P$ дискретных равноотстоящих значений, для каждого из которых вычислено значение целевой функции $\Phi_{p} ; p=1, \ldots, P$. Возьмем тройку соседних значений $\Phi_{p-1}, \Phi_{p}$ и $\Phi_{p+1}$, где $1<p<P$, и проверим выполнение неравенств:

$$
\begin{gathered}
\left(\Phi_{p+1}-\Phi_{p}\right)\left(\Phi_{p}-\Phi_{p-1}\right)<0, \\
\left(\Phi_{p+1}-2 \Phi_{p}+\Phi_{p-1}\right)>0 .
\end{gathered}
$$

Одновременное выполнение неравенств (8) и (9) указывает на то, что $\Phi_{p}$ является минимальным значением среди своих соседей $\Phi_{p-1}$ и $\Phi_{p+1}$. Аккумулируя такие $\Phi_{p}$, получим набор локальных минимумов целевой 
«Системні технологіï» 5 (124) 2019 «System technologies»

функции. Однако заметим, что для применения этого подхода к неискаженным данным заданный диапазон значений аргумента-параметра (например, межквартильный интервал) необходимо расширить для исключения возможности появления локальных минимумов на его границах.

Отбор основных локальных минимумов можно выполнить таким способом. Основными локальными минимумами будем считать наиболее глубокие локальные минимумы, которые превышают заданный порог глубины и расположены далеко друг от друга. Значение такого порога можно взять равным среднему арифметическому значению минимального и максимального значений целевой функции на заданной области значений аргумента-параметра. Если полученные в результате вычислений глубокие локальные минимумы окажутся ближе друг к другу, чем величина допустимого расстояния, то целесообразно считать, что сглаживание целевой функции было недостаточным и его следует повторить с увеличенным значением параметра $\alpha$.

Таким образом, предлагаемые методы одномерной минимизации функционала квазипротяженности заключаются в построении соответствующей целевой функции, априорном или апостериорном определении допустимого множества значений неизвестного параметра и поиске на нем всех локальных минимумов с последующим отбором основных локальных минимумов.

Рассмотрим задачу многомерной минимизации функционала квазипротяженности для случая нескольких линейных параметров, каждый из которых принимает единственное значение. В этом случае решение задачи заключается в поиске глобального минимума соответствующей целевой функции в многомерном пространстве, размерность которого равна количеству неизвестных параметров. Очевидно, что это решение требует привлечения более эффективных методов оптимизации, чем методы нулевого порядка. Одним из них является метод сопряженных градиентов, который обычно рекомендуется к использованию для минимизации нелинейных многомерных целевых функций в качестве 
«единственного подходящего универсального метода» [12]. Этот метод задается итерационной схемой:

$$
\begin{aligned}
& \mathbf{u}^{(t+1)}=\mathbf{u}^{(t)}+h^{(t)} \mathbf{p}^{(t)}, \quad t \geq 0 ; \\
& \mathbf{p}^{(0)}=-\mathbf{g}^{(0)}, \quad t=0 ; \quad \mathbf{p}^{(t)}=-\mathbf{g}^{(t)}+d^{(t-1)} \mathbf{p}^{(t-1)}, \quad t \geq 1 ; \\
& d^{(t-1)}=\left\|\mathbf{g}^{(t)}\right\|^{2} /\left\|\mathbf{g}^{(t-1)}\right\|^{2}, \quad h^{(t)}=\underset{h}{\arg \min \Phi\left(\mathbf{u}^{(t)}+h \mathbf{p}^{(t)}\right)}
\end{aligned}
$$

где $t$ - номер итерации; $\mathbf{u}^{(t)}$ - вектор решения на $t$-ой итерации; $h^{(t)}$ шаг вдоль направления спуска $\mathbf{p}^{(t)}$ на $t$-ой итерации; $\mathbf{g}^{(t)}$ - вектор градиента целевой функции Ф на $t$-ой итерации. Для применения (10) необходимо вычислять вектор градиента целевой функции Ф и решать одномерную задачу ее минимизации путем выбора величины шага вдоль направления спуска. Как отмечено выше, вычисление вектора градиента целевой функции возможно в случае, когда эта функция для заданных $\alpha$, $\beta, q$ является дифференцируемой (т.е. для $\alpha>0,-\infty<\beta<1$ и $1<q<\infty$ ). Однако даже в этом случае отдельные компоненты вектора градиента могут неверно указывать направление спуска. В связи с этим необходимо обеспечить такое решение одномерной задачи минимизации, которое будет приводить к уменьшению значения целевой функции за счет выбора величины шага вдоль сформированного направления спуска. Поэтому задачу выбора величины шага целесообразно решать методом нулевого порядка, который позволит явно контролировать уменьшение значения целевой функции и не допустит ее увеличения. Однако методы первого или второго порядка также могут быть применены в этой задаче с целью формирования некоторых пробных шагов, которые могут оказаться эффективными тогда, когда решение задачи окажется в такой окрестности минимума целевой функции, в которой целевая функция является выпуклой. Отсюда следует, что задачу одномерной минимизации целесообразно решать методом нулевого порядка на основе множества пробных шагов. В частности, в это множество можно включить шаги, которые минимизируют каждый элемент вектора невязки, а также шаг на основе метода Ньютона. Тогда алгоритм использования пробных шагов заключается в подстановке каждого из них в одномерную задачу минимизации и выборе в качестве $h^{(t)}$ такого значения шага, для которого Ф 
«Системні технологіï» 5 (124) 2019 «System technologies»

принимает минимальное значение. Если наилучшее значение шага минимизирует некоторый элемент невязки, то следует обновить направление спуска по формуле: $\mathbf{p}^{(t)}=-\mathbf{g}^{(t)}$. Если шаг, который отвечает методу Ньютона, принимает наилучшее значение $N$ раз подряд, то также следует обновить направление спуска. Если наилучшее значение шага равно нулю, то необходимо сформулировать вывод: " $\mathbf{u}^{(t)}$ есть решение задачи" и остановить итерационный процесс.

На рис.1 приведен пример численного моделирования одномерной задачи минимизации функционала квазипротяженности для случая линейного параметра, которым являлась амплитуда синусоиды. Исходная зависимость (рис. 1а) описывалась соотношением:

$$
g(x)=\left\{\begin{array}{cc}
\sin \left(2 \pi v x+\varphi_{0}\right)+\xi ; & x \in[0 ; 2,5) \\
2 \sin \left(2 \pi v x+\varphi_{0}\right)+\xi ; & x \in[2,5 ; 5]
\end{array},\right.
$$

где $v=1 ; \varphi_{0}=\pi / 8$, и была задана с шагом $\Delta x=0,01$ на $N=501$ точке, включая концы интервала. Случайная величина $\xi$ была распределена по закону Коши: $p(\xi)=(v / \pi) \cdot\left(\xi^{2}+v^{2}\right)^{-1}$ с параметром масштаба $v=0,1$ и нулевым параметром сдвига. Модель синусоиды задавалась формулой: $s(x)=A f(x)=A \sin \left(2 \pi v x+\varphi_{0}\right)$; ее форма $f(x)$ представлена на рис. 1б. Целевая функция функционала квазипротяженности была построена для значений $\alpha=0,1 ; \beta=0 ; q=2$. Поиск и отбор основных локальных минимумов целевой функции выполнялся на расширенном межквартильном интервале [0,978; 2,057], который был вычислен на основе описанной выше модифицированной версии способа выбора допустимой области значений неизвестного параметра. Видно (рис. 1в), что найденные в точках $A=1,075$ и $A=1,949$ локальные минимумы являются близкими к искомым, отвечая истинным значениям амплитуды в (11) с точностью, которая примерно равна величине параметра сглаживания $\alpha=0,1$. 
«Системні технологіï» 5 (124) 2019 «System technologies»

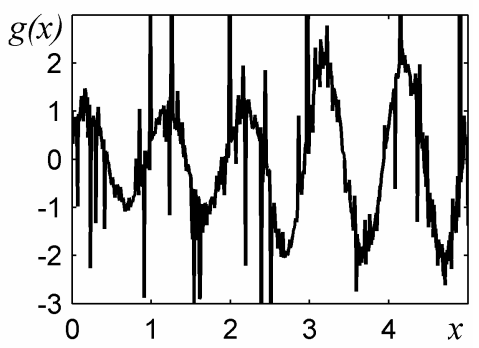

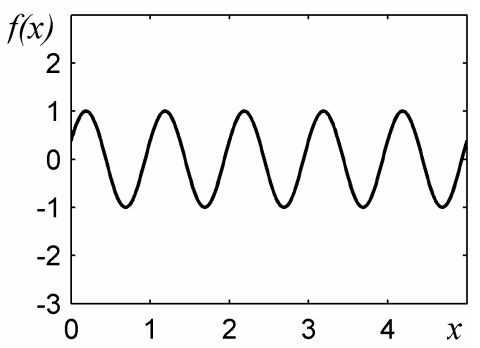

a

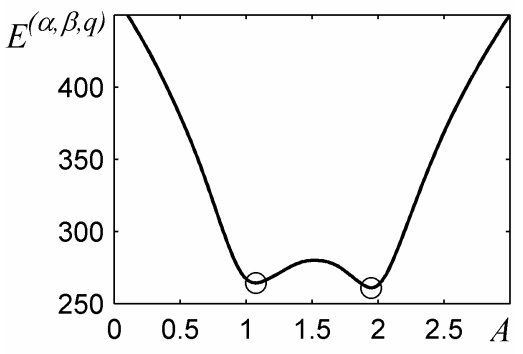

B

Рисунок 1 - Моделирование для линейного параметра: а - исходная зависимость; б - модель; в - целевая функция; найденные минимумы отмечены кружками.

На рис.2 приведен пример численного моделирования одномерной задачи минимизации функционала квазипротяженности для случая нелинейного параметра, которым являлась частота синусоиды. Исходная зависимость (рис. 2а) описывалась соотношением:

$$
g(x)=\left\{\begin{array}{ll}
\sin \left(2 \pi v_{1} x+\varphi_{0}\right)+\xi ; & x \in[0 ; 2,5) \\
\sin \left(2 \pi v_{2} x+\varphi_{0}\right)+\xi ; & x \in[2,5 ; 5]
\end{array},\right.
$$

где $v_{1}=1 ; v_{2}=4 ; \varphi_{0}=\pi / 8$, и была задана с шагом $\Delta x=0,01$ на $N=501$ точке, включая концы интервала. Случайная величина $\xi$ была распределена по закону Коши с параметром масштаба $v=0,1$ и нулевым параметром сдвига. Модуль спектра $|G(v)|$ зависимости $g(x)$ приведен на рис. 2б, где видно, что идентификация искомых спектральных линий затруднена. Целевая функция функционала квазипротяженности была построена для тех же значений $\alpha=0,1 ; \beta=0 ; q=2$. Поиск и отбор основных локальных минимумов выполнялся на основе априорно заданных (аналогично дискретному преобразованию Фурье) значений интервала частот [0;50] и шага по частоте $\Delta v=1 /((N-1) \cdot \Delta x)=0,2$. Поскольку $v_{1}=1$ и $v_{2}=4$, то эти искомые значения частоты попадали в шестой и 21-й узлы построенной сетки частот. На рис. 2в видно, что найденные локальные минимумы верно отвечают истинным значениям частоты в (12), имея точность, равную величине шага $\Delta v$. 
«Системні технологіï» 5 (124) 2019 «System technologies»

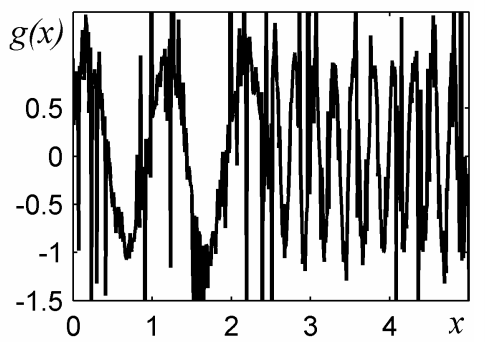

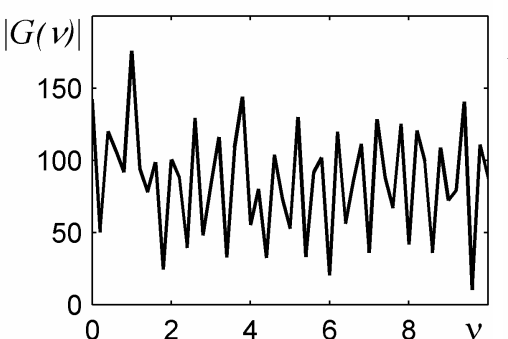

a

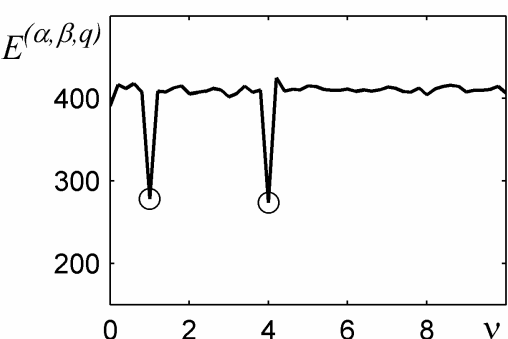

B

Рисунок 2 - Моделирование для нелинейного параметра: а - исходная зависимость; б - модуль спектра; в - целевая функция, на которой найденные минимумы отмечены кружками.

Численное моделирование задачи многомерной минимизации функционала квазипротяженности выполнялось на примере задачи решения переопределенной комплекснозначной системы линейных алгебраических уравнений (СЛАУ), правая часть которой содержала шум и грубые ошибки [1]. Результаты этого моделирования представляют качество решения задачи определения параметров дипольных источников излучения, которые были расположены в точках $x / \lambda$, равных $3,4,5,7$ и 8. На рис. За в виде графиков отображены реальная (сплошная кривая) и мнимая (пунктирная кривая) части комплексных значений правой части

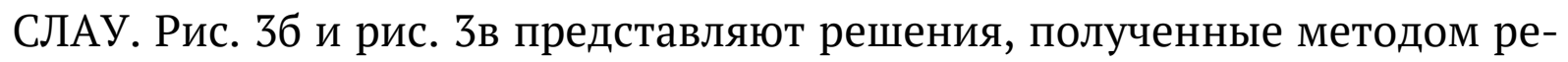
гуляризации Тихонова и методом (10), соответственно (сплошные кривые), а также решение методом регуляризации Тихонова при отсутствии грубых ошибок (пунктирная кривая). При этом решение на основе (10) дало меньшую ошибку и визуально является более близким к искомому.
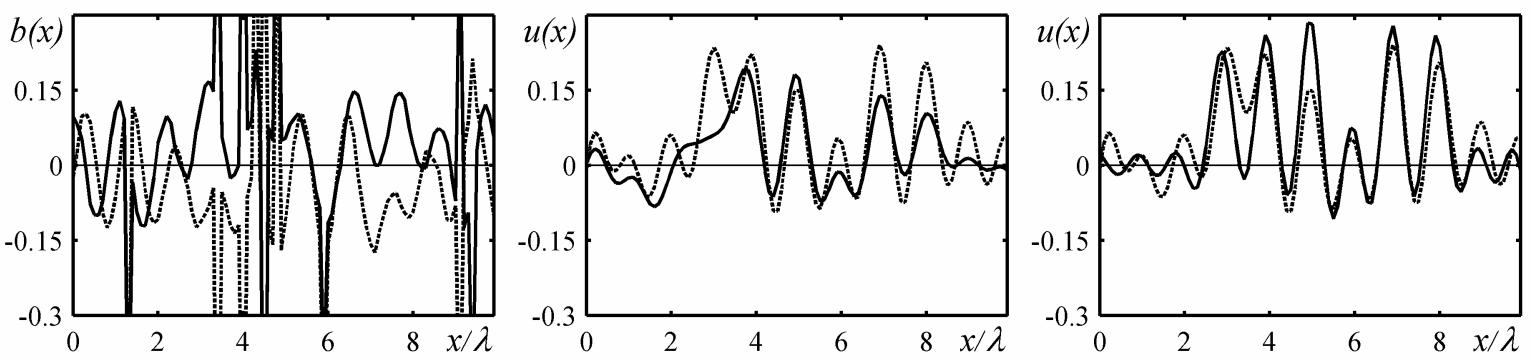

a

6

B

Рисунок 3 - Моделирование для нескольких линейных параметров: а данные; б - решение методом Тихонова; в - решение на основе (10). 
«Системні технологіï» 5 (124) 2019 «System technologies»

Выводы. Предложенные методы численной минимизации функционала квазипротяженности обеспечивают численное решение задач обработки данных, которые сформулированы на основе критерия минимума протяженности в виде задач минимизации функционала квазипротяженности. Методы одномерной минимизации реализованы на основе методов оптимизации нулевого порядка и позволяют решать задачи определения значений одного неизвестного параметра для случаев, когда он входит в модель данных линейно или нелинейно и принимает одно или несколько значений. Метод многомерной минимизации реализован на основе метода сопряженных градиентов и позволяет решать задачи определения значений нескольких неизвестных линейных параметров, каждый из которых принимает одно значение. Результаты численного моделирования подтверждают эффективность применения предложенных методов.

\section{ЛИТЕРАТУРА / ЛІТЕРАТУРА}

1. Вовк С.М. Постановка задач обработки данных на основе критерия минимума протяженности / С. М. Вовк // Радіоелектроніка, інформатика, управління. - 2019. - N.1 -C. 157-166. DOI: 10.15588/1607-3274-2019-1-15.

2. Вовк С.М. Критерій мінімуму протяжності / С. М. Вовк // Системні технології. Регіональний міжвузівський збірник наукових праць. Випуск 1 (120). - Дніпро, 2019. - С. 19 - 25.

3. Вовк С.М. Метод обробки даних в умовах складного шумового оточення / С.М. Вовк, В. В. Гнатушенко // Науковий вісник Таврійського державного агротехнологічного університету. - 2018. - Вип. 8. - Т.2. - С. 1-9. DOI: $10.31388 / 2220-8674-2018-2$.

4. Titchmarsh E. C. The theory of functions / E. C. Titchmarsh. - New York: Oxford Press, 1939. - 454 p.

5. Михлин С.Г. Вариационные методы в математической физике / С. Г. Михлин. - М.: Наука, 1970. - 512 с.

6. Васильев Ф.П. Численные методы решения экстремальных задач / Ф.П. Васильев. - М.: Наука, 1988. - 552 с. 
«Системні технології» 5 (124) 2019 «System technologies»

7. Gonzalez J.G. Weighted myriad filters: a robust filtering framework derived from alpha-stable distributions / J. G. Gonzalez, G. R. Arce // Proc. of Int. Conf. Acoustic, Speech and Signal Processing, Atlanta, 1996. - P. 2833-2836.

8. Aysal T.C. Meridian filtering for robust signal processing / T.C. Aysal, K.E. Barner // IEEE Tr. Signal Processing. - 2007. - V. 55. - N. 8. - P. 3949-3962.

9. Вовк С.М. Декомпозиция суммы гауссиан, искаженных импульсным шумом / С.М. Вовк // Радіоелектроніка, інформатика, управління. - 2017. - N. 1 - C.91-99. DOI:10.15588/1607-3274-2017-1-11.

10. Грановский В. А. Методы обработки экспериментальных данных при измерениях / В. А. Грановский, Т. Н. Сирая. - Л.: Энергоатомиздат, 1990. -288 c.

11. Бейко И.В. Методы и алгоритмы решения задач оптимизации / И. В. Бейко, Б. Н. Бублик, П. Н. Зинько. - К.: Вища школа, 1983 .- 512 с.

12. Амосов А.А. Вычислительные методы решения инженерных задач. Приближение функций, численное интегрирование, минимизация функций / А.А. Амосов, Ю.А. Дубинский, Н.В. Копченова.- М.: Изд-во МЭИ, 1992.- 192 c.

\section{REFERENCES}

1. Vovk S.M. Postanovka zadach obrabotki dannyih na osnove kriteriya minimuma protyazhennosti / S. M. Vovk // Radioelektronika, informatyka, upravlinnia. - 2019. - N.1 - S. 157-166. DOI: 10.15588/1607-3274-2019-1-15.

2. Vovk S.M. Kryterii minimumu protiazhnosti / S. M. Vovk // Systemni tekhnolohii. Rehionalnyi mizhvuzivskyi zbirnyk naukovykh prats. Vypusk 1 (120). - Dnipro, 2019. - C. 19 - 25.

3. Vovk S.M. Metod obrobky danykh v umovakh skladnoho shumovoho otochennia / S.M. Vovk, V. V. Hnatushenko // Naukovyi visnyk Tavriiskoho derzhavnoho ahrotekhnolohichnoho universytetu. - 2018. - Vyp. 8. - T.2. - S. 19. DOI: $10.31388 / 2220-8674-2018-2$.

4. Titchmarsh E. C. The theory of functions / E. C. Titchmarsh. - New York: Oxford Press, 1939. - $454 \mathrm{p}$.

5. Mihlin S.G. Variatsionnyie metodyi v matematicheskoy fizike / S. G. Mihlin. - M.: Nauka, 1970. - 512 s. 
6. Vasilev F.P. Chislennyie metodyi resheniya ekstremalnyih zadach / F. P. Vasilev. - M.: Nauka, 1988. - 552 s.

7. Gonzalez J.G. Weighted myriad filters: a robust filtering framework derived from alpha-stable distributions / J. G. Gonzalez, G. R. Arce // Proc. of Int. Conf. Acoustic, Speech and Signal Processing, Atlanta, 1996. - P. 2833-2836.

8. Aysal T. C. Meridian filtering for robust signal processing / T. C. Aysal, K. E. Barner // IEEE Tr. Signal Processing. - 2007. - V. 55. - N. 8. - P. 3949-3962.

9. Vovk S.M. Dekompozitsiya summyi gaussian, iskazhennyih impulsnyim shumom / S. M. Vovk // Radioelektronika, informatyka, upravlinnia. - 2017. N. 1 - S.91-99. DOI:10.15588/1607-3274-2017-1-11.

10. Granovskiy V.A. Metodyi obrabotki eksperimentalnyih dannyih pri izmereniyah / V.A. Granovskiy, T.N. Siraya. - L.:Energoatomizdat, 1990. -288 s.

11. Beyko I.V. Metodyi i algoritmyi resheniya zadach optimizatsii / I. V. Beyko, B.N. Bublik, P. N. Zinko. - K.: Vischa shkola, 1983 .- 512 s.

12. Amosov A.A. Vyichislitelnyie metodyi resheniya inzhenernyih zadach.

Priblizhenie funktsiy, chislennoe integrirovanie, minimizatsiya funktsiy / A.A. Amosov, Yu.A. Dubinskiy, N. V. Kopchenova.- M.: Izd-vo MEI, 1992.- 192 s.

Received 09.10.2019. Accepted 11.10.2019.

Методи чисельної мінімізації функціоналу квазіпротяжності

Розглядаються методи чисельної мінімізації функціоналу квазіпротяжності, який побудований на основі функції відхилу даних від їх моделі. Ці методи складаються з методів одновимірної мінімізації за невідомим параметром моделі даних, який входить в модель даних лінійно або нелінійно та приймає одне або декілька значень, та з методу багатовимірної мінімізації за декількома невідомими лінійними параметрами моделі, кожен з яких приймає одне значення. Методи одновимірної мінімізації засновані на методах оптимізації нульового порядку. Метод багатовимірної мінімізації заснований на методі спряжених градієнтів. Наведені приклади чисельного моделювання, які демонструють ефективність використання запропонованих методів.

\section{Numerical minimization methods for functional of quasi-extent}

The data processing problems formulation based of the minimum-extent criterion leads to the problems of minimizing the functional of quasi-extent. Due to the impossibility of using analytical methods of calculus of variations, the successful solutions of these problems depend on the effectiveness of numerical minimization methods. In this paper, the problem of developing methods for one-dimensional and multidimensional numerical minimization of the quasi-extent functional, which is built on the residual function between the data and their model, is considered. The goal of this work is to create the effective methods for numerically minimizing the quasi-extent functional.

In accordance to the problem statement, the methods of one-dimensional and multidimensional numerical minimization are considered. The main requirements to the methods of one-dimensional minimization of the quasi-extent functional were consisted both in the strict 


\section{«Системні технології» 5 (124) 2019 «System technologies»}

fulfillment of the condition for non-increasing of objective function formed on the basis of the quasi-extent functional as well as in the fulfillment of the condition of searching the several local minima. For this reason, the proposed one-dimensional minimization methods were based on the zero-order optimization methods with a priori or a posteriori choice of the feasible region of the objective function and with a selection of the set of basic local minima. The results of numerical simulation showed that the proposed methods allow solving successfully the problem of determining the values of single unknown data model parameter which is included in the data model linearly or nonlinearly and accepted one or more values. The main requirements to the methods of multidimensional minimization of the quasi-extent functional, which is depended on several unknown linear parameters, were consisted both in the fulfilling of the condition for nonincreasing of objective function formed on the basis of the quasi-extent functional, as well as in the fulfillment of the condition of searching the global minimum. Due to these requirements, the proposed multidimensional minimization method was based on the conjugate gradient method, in which the one-dimensional minimization problem along the descent direction is solved by using the set of "test" steps. The results of numerical simulation confirmed the performance of proposed method.

Вовк Сергей Михайлович - к.ф.-м.н., доцент кафедры компьютерных наук и информационных технологий Днепровского национального университета им. Олеся Гончара.

Вовк Сергій Михайлович - к.ф.-м.н., доцент кафедри комп’ютерних наук та інформаційних технологій Дніпровського національного університету ім. Олеся Гончара.

Vovk Serhii - Ph.D., Associate Professor, Department of Computer Science and Information Technology, Oles Honchar Dnipro National University. 